\title{
ACESSO ABERTO, REPOSITÓRIOS, PORTAIS DE PUBLICAÇões, PRESERVAÇÃo E COMPORTAMENTO INFORMACIONAL NO CONTEXTO ACADÊMICO
}

${ }^{1}$ Gildenir Carolino Santos

Danielle Thiago Ferreira

Universidade Estadual de Campinas ${ }^{1}$

Correspondência

Gildenir Carolino Santos

Universidade Estdaual de Campinas,

Sistema de Bibliotecas

Campinas - SP - Brasil.

Email: gilldenir@unicamp.br

ORCID: https://orcid.org/0000-0002-4375-6815

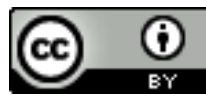


Prezados Leitores,

É com prazer que lançamos mais uma edição da RDBCI, volume16, número 2, de maio a agosto de 2017, que traz como temática para essa edição o "Acesso aberto, repositórios, portais de publicações, preservação e comportamento informacional no contexto acadêmico".

Nesta edição teremos uma seção especial intitulada Dossiê, onde o objetivo é apresentar trabalhos advindos da Conferencia Internacional Biredial - ISTEC 2017, que aconteceu na cidade de La Plata na Argentina, nos dias 2, 3 e 4 de outubro de 2017. Este evento foi promovido pela Universidade Nacional de La Plata, Comissão de Investigações Científicas da Argentina e Consorcio Iberoamericano para Educação Ciência e Tecnologia (ISTEC). Teve como colaboradores a Universidade do Rosário da Colômbia, Universidade de Costa Rica, Universidade Autônoma de São Luís Potosi do México e Universidade Federal do Rio Grande do Sul, Brasil.

A Conferência Internacional BIREDIAL - ISTEC dedica-se a discutir, analisar e promover iniciativas institucionais, nacionais e regionais relacionadas com o acesso aberto e a visibilidade das pesquisas e publicações acadêmicas latino-americanas. $\mathrm{O}$ foco deste evento de 2017, também foi discutir cenários sobre a gestão de dados de pesquisa para acesso público e a avaliação e certificação dos repositórios acadêmicos institucionais; assuntos com muito destaque hoje no mundo acadêmico.

Foram selecionais quatro trabalhos para publicação na revista previamente avaliados pela Comissão Organizadora do Evento em parceria com a Comissão Editorial da RDBCI; que esteve presente na ocasião do evento.

Iniciamos esta seção com o trabalho de Marisa R. De Giusti e Gonzalo L. Vilareal, responsáveis pelo Programa de Intercâmbio de Serviço de Difusão e criação intelectual e pelo Centro de Serviços em gestão da Informação da Universidade de La Plata. O trabalho intitulado "Revisão de diferentes implementações para preservação digital: uma proposta metodológica para a preservação e auditoria de confiabilidade do Repositório Institucional" tem como objetivo relatar a experiência de implantar uma estrutura adequada para a preservação de documentos digitais em um arquivo ou repositório. São descritas as iniciativas existentes hoje na literatura sobre preservação de conteúdo digital para repositórios e depois relata a iniciativa e estudos sobre os repositórios gerenciados no PrEBi-SEDICI (UNLP) com DSpace, Archivematica e ArchivesSpace. A grande contribuição deste trabalho é o fato de registrar as iniciativas de preservação com o objetivo de ser uma fonte de revisão para o contexto latino-americano, onde essas questões são incipientes.

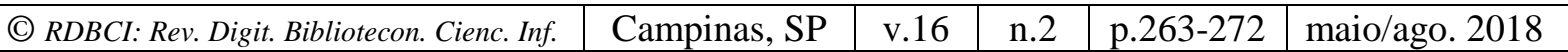


O próximo trabalho desta seção intitula-se "Análise dos sistemas de busca de revistas científicas eletrônicas”, de Paula Caroline Schifino Jardim Passos e Sônia Elisa Caregnato, pesquisadora e professora da Universidade Federal do Rio Grande do Sul. Este trabalho analisa o sistema de busca em três revistas de renome internacional Nature, Science e PLOS Biology. Para tanto, o método escolhido foi a inspeção por lista de verificação, que por meio de verificação sistemática e aprofundada permitiu elencar os recursos aplicados à busca e recuperação de informação em revistas científicas de alta visibilidade. Por outro lado, foi possível identificar pontos onde algumas modificações trariam benefícios à experiência dos usuários das revistas. Além disso, a comparação entre os três títulos, evidenciou as melhores soluções utilizadas em cada um dos mecanismos de busca, considerando a importância de constantes estudos para atualizações tecnológicas para as revistas eletrônicas científicas promovendo a visibilidade das pesquisas ali publicadas.

O próximo trabalho da seção Dossiê, de autoria de Marcela Fushimi, Mariana Pichinini, Ana María Sanllorenti e Martín Williman (professores, bibliotecários e pesquisadores da Universidade Nacional de La Plata e Universidade de Buenos Aires), intitulado "Greenstone: uso actual en Argentina", apresenta as características e a história do software "Greenstone" gerenciador de bibliotecas digitais, desenvolvido inicialmente pela Universidade de Waikato, Nova Zelândia, em 1997. Os dados coletados das pesquisas permitiram observar que os motivos predominantes para a escolha desta plataforma, que seriam facilidade de instalação e configuração, baixo nível de requisitos tecnológicos, etc.; e a evolução para uma nova versão em 2016, implementando uma reengenharia completa do software para adaptá-la às tecnologias de uso atual (XML, XSLT, serviços Web e Java).

O último trabalho desta seção é o de Caterina Groposo Pavão, Rafael Porte da Rocha e Rene Faustino Gabriel Junior (bibliotecárias e professores da Universidade Federal do Rio Grande do Sul) da que trata da "Proposta de criação de uma rede de dados abertos da pesquisa brasileira", que aborda a necessidade de tornar público os dados de pesquisa resultantes de investigações realizadas em instituições de ensino e pesquisa brasileiras, por meio do acesso aberto em repositórios. O objetivo desta iniciativa é de validar os resultados obtidos e publicados, como também impulsionar novas pesquisas e socializar o conhecimento. $\mathrm{O}$ trabalho explora o cenário nacional e internacional e apresenta o planejamento de uma investigação que busca uma solução tecnológica para efetivar o acesso aberto aos dados de pesquisa (AADP) dentro de uma perspectiva nacional baseado no levantamento dos serviços e soluções tecnológicas para o compartilhamento de dados de pesquisa existentes no cenário internacional e faz a recomendações da criação de repositórios de dados de pesquisa em instituições nacionais e a sua agregação a uma rede de pesquisa em AADP.

Dando continuidade aos trabalhos focados no fluxo contínuo da RDBCI, iremos apresentar oito artigos, dois artigos de pesquisas e uma resenha. 
Na seção Artigos, o primeiro trabalho intitulado "Aspectos epistemológicos da ciência da informação e o comportamento informacional: diálogos com Borko, Le Coadic e Saracevic”, Jorge Santa Anna (Universidade Federal do Espírito Santo) comenta que os estudos sobre usuários e seus comportamentos no que tange à busca por informação tem demonstrado o quanto o aspecto humano-social tem sido valorizado pelas ciências, sobretudo na Ciência da Informação. Portanto, o autor apresenta uma pesquisa preliminar, cujo objetivo é discorrer alguns aspectos epistemológicos da Ciência da Informação, bem como os estudos do comportamento informacional, tendo como base teórica as reflexões apresentadas por Borko, Le Coadic e Saracevic. Por meio de pesquisa descritiva e bibliográfica de abordagem dedutiva, o autor confirmou que a Ciência da Informação estuda o objeto informação e os procedimentos para coleta, armazenagem e uso desse recurso, caracterizando-se como uma ciência interdisciplinar, influenciada pelas tecnologias da informação e comunicação, cujas origens desse campo científico remetem aos reflexos do desenvolvimento científico e tecnológico do século XX. Além disso, concluiu-se que os estudos das necessidades e do uso da informação, com foco no usuário, viabiliza a importância do ser humano, como principal elemento do processo informacional, sendo que os estudos vêm se aperfeiçoando, em que são investigadas questões comportamentais quanto ao uso dos recursos, produtos, serviços e sistemas de informação.

No segundo artigo sobre "A repercussão de artigos de periódicos brasileiros da ciência da informação no Facebook: um estudo altmétrico”, Ronaldo Ferreira Araújo (Universidade Federal de Alagoas), Tiago Rodrigo Marçal Murakami (Universidade de São Paulo) e Jorge Moisés Kroll do Prado (Rede de Bibliotecas do SENAC-SC) refletem sobre as interações do Facebook enquanto elementos de atenção online e fonte de dados altmétricos, e dialoga as reflexões com uma análise empírica que considerou a repercussão de artigos de periódicos da Ciência da Informação indexados no Repertório da Produção Periódica Brasileira de Ciência da Informação (RPPBCI). A coleta de dados altmétricos foi realizada por meio da utilização de Application Program Interface do Facebook parametrizada pela URL principal e secundária de artigos de 31 periódicos.

Na sequência, Jovenilda Freitas dos Santos (Universidade Federal de Sergipe) e Nídia Maria Lienert Lubisco (Universidade Federal da Bahia) no trabalho intitulado " $O$ desenvolvimento do marketing e sua aproximação com os paradigmas da Ciência da Informação", as autoras discutem, sob uma perspectiva histórica, o desenvolvimento do Marketing e sua convergência com os paradigmas da Ciência da Informação, conhecidos como paradigma físico, cognitivo e social. Sob o paradigma físico, as autoras pontuam a relação do nascimento de ambas as áreas do conhecimento, que exclui o papel ativo do sujeito cognoscente de todo processo informativo e comunicativo. Em seguida, elas, sob a visão cognitiva, analisam a proximidade das áreas, a partir das interações e necessidades do usuário em relação aos sistemas de informação, onde ambas as áreas não tinham como compreender o usuário, de modo a ajustar os serviços a ele.

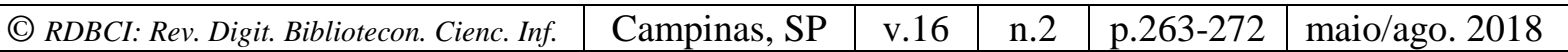


Em "Folksonomia em sites de redes sociais segmentadas (SRSS) em livros: um estudo exploratório da interface do Goodreads", Adriana Amaral (UNISINOS) e Tarciso Salvador (Universidade Federal do Rio Grande do Sul) mapeiam e discutem os conceitos em torno da folksonomia e do etiquetamento em Sites de Redes Sociais Segmentadas em livros, levando em consideração as diferentes formas de classificação e categorização feitas pelos usuários do site Goodreads.

No quinto artigo, Tatiane Priscila Fortunato e Adilson Luiz Pinto (Universidade Federal de Santa Catarina) sob o trabalho intitulado "Análise das informações científicas utilizadas na gestão da informação das instituições de ensino superior segundo a literatura internacional”, referem-se ao estudo da Gestão da Informação, no intuito de enfatizar sua relevância para a acessibilidade da informação e o desenvolvimento e disseminação do conhecimento nas diversas áreas de conhecimento.

No artigo seguinte sobre " $O$ silenciamento do multiculturalismo na pauta das Universidades Federais do sudeste e nordeste brasileiro: tendências na formação do bibliotecário e cientista da informação”, os autores Claudio Moises da Costa e Patrícia Vargas Alencar (Universidade Federal do Estado do Rio de Janeiro) investigam a formação do bibliotecário e do cientista da informação nas universidades federais das regiões Sudeste e Nordeste do Brasil. Os autores discutem até que ponto os cursos de Biblioteconomia e Ciência da Informação contemplam o Multiculturalismo no âmbito do ensino, da pesquisa e da extensão universitária.

No trabalho "A mediação da informação na narrativa oral e na história de vida: proposições dialogais”, Ana Cristina Guimarães Carvalho (Universidade Federal do Piauí), Maria Gezilda e Silva Nascimento (Instituto Federal de Educação, Ciência e Tecnologia do Maranhão), Midinai Gomes Bezerra (Universidade Estadual da Paraíba) se propõem a discutir a mediação da informação e suas relações com a narrativa oral e com a história de vida, enquanto potenciais recursos informacionais para a apropriação e construção do conhecimento. Para tanto, os autores tiveram como problematização norteadora a seguinte indagação: quais as possíveis relações entre a mediação da informação, a narrativa oral e a história de vida no âmbito do fazer bibliotecário? Nesse sentido, os autores objetivam a abordar as concepções de mediação da informação a partir do fazer bibliotecário, visando uma aproximação com as perspectivas da narrativa oral e história de vida.

No oitavo e último artigo trazemos Sonia Boeres (Universidade de Brasília) falando sobre " $O$ letramento e a organização da informação digital aliados ao aprendizado ao longo da vida". A autora diz que o letramento digital é fruto de pensamento crítico e busca potencializar os métodos de estudo pela tecnologia da informação. O objetivo da pesquisa é compreender como o letramento digital pode contribuir para o aprendizado ao longo da vida. Com isso, a

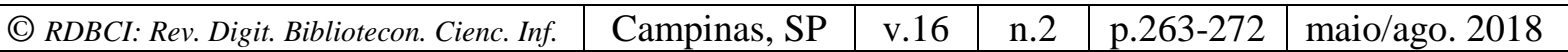


autora fez uma revisão de literatura, nacional e internacional, sobre o processo de ensino e aprendizagem, letramento informacional, digital e aprendizado ao longo da vida para metodologicamente descrever a pesquisa.

Na seção Artigos de Pesquisa trazemos o primeiro trabalho intitulado "Conexão entre competência em informação e as disciplinas fontes de informação e serviço de referência: um mapa conceitual", de autoria de Elisa Cristina Delfini Correa, Elaine Rosangela de Oliveira Lucas e Viviane Formighieri Muller (Universidade do Estado de Santa Catarina) que realizam a análise das possibilidades teóricas e práticas da aplicação de modelos de competência em informação nas disciplinas de Fontes de Informação e Serviço de Referência, com base em seus planos de ensino. As autoras com base na pesquisa documental realizam uma análise comparativa entre a literatura sobre o tema e os planos de ensino das disciplinas de Fontes de Informação e Serviço de Referência, oferecidas no curso de graduação em Biblioteconomia da Universidade do estado de Santa Catarina. Os resultados da análise documental apontam para uma estreita relação entre as disciplinas e o modelo de competência em informação escolhido como base teórica em especial com a disciplina de Fontes de Informação. Sua aplicação oportuniza um aprendizado capaz de levar o future bibliotecário a atuar como mediador no processo de desenvolvimento de habilidades para a busca, acesso, assimilação da informação a fim de solucionar problemas e de construir um ambiente de geração e transmissão de conhecimentos junto à comunidade de sua unidade de informação. Apresenta ao final um mapa conceitual desenvolvido a partir da pesquisa bibliográfica e documental.

O segundo artigo de pesquisa sobre os "Websites em bibliotecas universitárias como instrumentos para a formação de competências em informação", Marcos Aurélio Gomes (Universidade Federal de Alagoas) e Lígia Maria Moreira Dumont (Universidade Federal de Minas Gerais) objetivam identificar nos websites das bibliotecas universitárias a prática da educação/formação de usuários por meio de produtos e serviços que podem ser fomentados tanto em ambientes tradicionais como digitais/virtuais na perspectiva da promoção de competências em informação dos estudantes. Os autores, que classificaram a pesquisa como exploratória, estabeleceram em seu desenho como recorte duas universidades: a Universidade Federal de Alagoas e a Universidade Federal de Minas Gerais. A análise dos dados foi realizada com enfoque quantitativo e qualitativo. Adotou-se como instrumento de coleta o formulário, que proporcionou o levantamento de dados em 35 bibliotecas das duas instituições. Os resultados forneceram evidências de que as bibliotecas das duas universidades encontram-se em processos de transição considerando o ponto de vista de uso da tecnologia digital para a promoção das ações de educação/formação de usuário para promover a competência em informação.

Na última seção Resenha desta edição Jacqueline Messias dos Santos e Gildenir Carolino Santos (Universidade Estadual de Campinas) apresentam a resenha da obra "Como não \begin{tabular}{l|l|l|l|l|l} 
(C) RDBCI: Rev. Digit. Bibliotecon. Cienc. Inf. & Campinas, SP & v.16 & n.2 & p.263-272 & maio/ago. 2018 \\
\hline
\end{tabular} 
escrever um artigo médico: um guia prático", escrita por Markus K. Heinemann, editor-chefe do periódico "The Thoracic and Cardiovascular Surgeon", da Sociedade Alemã de Cirurgia Torácica e Cardiovascular, de Mainz, Alemanha.

Assim, finalizamos mais uma edição. Tenham todos, uma boa leitura dos preciosos trabalhos que aqui apresentamos e até a próxima!

Gildenir Carolino Santos

Danielle Thiago Ferreira Editores Científicos - RDBCI

Abril / 2018.

\section{REFERÊNCIAS}

ARAUJO, Ronaldo Ferreira; MURAKAMI, Tiago Rodrigo Marçal; PRADO, Jorge Moisés Kroll do. A repercussão de artigos de periódicos brasileiros da ciência da informação no Facebook: um estudo altmétrico. RDBCI: Revista Digital de Biblioteconomia e Ciência da Informação, Campinas, SP, v. 16, n. 2, jan. 2018. ISSN 1678-765X. Disponível em: <https://periodicos.sbu.unicamp.br/ojs/index.php/rdbci/article/view/8650461 >. Acesso em: 26 abr. 2018. doi:https://doi.org/10.20396/rdbci.v16i2.8650461.

BOERES, Sonia. O letramento e a organização da informação digital aliados ao aprendizado ao longo da vida. RDBCI: Revista Digital de Biblioteconomia e Ciência da Informação, Campinas, SP, v. 16, n. 2, mar. 2018. ISSN 1678-765X. Disponível em: <https://periodicos.sbu.unicamp.br/ojs/index.php/rdbci/article/view/8651507>. Acesso em: 26 abr. 2018. doi:https://doi.org/10.20396/rdbci.v16i2.8651507.

CARVALHO, Ana Cristina Guimarães; NASCIMENTO, Maria Gezilda e Silva; BEZERRA, Midinai Gomes. A mediação da informação na narrativa oral e na história de vida: proposições dialogais. RDBCI: Revista Digital de Biblioteconomia e Ciência da Informação, Campinas, SP, v. 16, n. 2, mar. 2018. ISSN 1678-765X. Disponível em: <https://periodicos.sbu.unicamp.br/ojs/index.php/rdbci/article/view/8651516>. Acesso em: 26 abr. 2018. doi:https://doi.org/10.20396/rdbci.v16i2.8651516.

CORREA, Elisa Cristina Delfini; LUCAS, Elaine Rosangela de Oliveira; MULLER, Viviane Formighieri. Conexão entre competência em informação e as disciplinas fontes de informação e serviço de referência: um mapa conceitual. RDBCI: Revista Digital de Biblioteconomia e Ciência da Informação, Campinas, SP, v. 16, n. 2, jan. 2018. ISSN 1678-765X. Disponível em: <https://periodicos.sbu.unicamp.br/ojs/index.php/rdbci/article/view/8649760>. Acesso em: 26 abr. 2018. doi:https://doi.org/10.20396/rdbci.v16i2.8649760.

COSTA, Claudio Moises da; ALENCAR, Patricia Vargas. O silenciamento do multiculturalismo na pauta das Universidades Federais do sudeste e nordeste brasileiro: tendências na formação do bibliotecário e cientista da informação. RDBCI: Revista Digital \begin{tabular}{l|l|l|l|l|l} 
(C) RDBCI: Rev. Digit. Bibliotecon. Cienc. Inf. & Campinas, SP & v.16 & n.2 & p.263-272 & maio/ago. 2018
\end{tabular} 
de Biblioteconomia e Ciência da Informação, Campinas, SP, v. 16, n. 2, fev. 2018. ISSN 1678-765X. Disponível em:

<https://periodicos.sbu.unicamp.br/ojs/index.php/rdbci/article/view/8649938>. Acesso em: 26 abr. 2018. doi:https://doi.org/10.20396/rdbci.v16i2.8649938.

DE GIUSTI, Marisa Raquel; LUJÁN VILLARREAL, Gonzalo. Revisão de diferentes implementações para a preservação digital: para uma proposta metodológica de preservação e auditoria de confiança de RI. RDBCI: Revista Digital de Biblioteconomia e Ciência da Informação, Campinas, SP, v. 16, n. 2, abr. 2018. ISSN 1678-765X. Disponível em: <https://periodicos.sbu.unicamp.br/ojs/index.php/rdbci/article/view/8651589>. Acesso em: 26 abr. 2018. doi:https://doi.org/10.20396/rdbci.v16i2.8651589.

FORTUNATO, Tatiane Priscila; PINTO, Adilson Luiz. Análise das informações científicas utilizadas na gestão da informação das instituições de ensino superior segundo a literatura internacional. RDBCI: Revista Digital de Biblioteconomia e Ciência da Informação, Campinas, SP, v. 16, n. 2, jan. 2018. ISSN 1678-765X. Disponível em: <https://periodicos.sbu.unicamp.br/ojs/index.php/rdbci/article/view/8650974>. Acesso em: 26 abr. 2018. doi:https://doi.org/10.20396/rdbci.v16i2.8650974.

FUSHIMI, Marcela et al. Greenstone: uso atual na Argentina. RDBCI: Revista Digital de Biblioteconomia e Ciência da Informação, Campinas, SP, v. 16, n. 2, abr. 2018. ISSN 1678-765X. Disponível em: <https://periodicos.sbu.unicamp.br/ojs/index.php/rdbci/article/view/8651806>. Acesso em: 26 abr. 2018. doi:https://doi.org/10.20396/rdbci.v16i2.8651806.

GOMES, Marcos Aurelio; DUMONT, Lígia Maria Moreira. Websites em bibliotecas universitárias como instrumentos para a formação de competências em informação. RDBCI: Revista Digital de Biblioteconomia e Ciência da Informação, Campinas, SP, v. 16, n. 2, fev. 2018. ISSN 1678-765X. Disponível em: <https://periodicos.sbu.unicamp.br/ojs/index.php/rdbci/article/view/8650816>. Acesso em: 26 abr. 2018. doi:https://doi.org/10.20396/rdbci.v16i2.8650816.

PASSOS, Paula Caroline Schifino Jardim; CAREGNATO, Sônia Elisa. Análise dos sistemas de busca de revistas científicas eletrônicas. RDBCI: Revista Digital de Biblioteconomia e Ciência da Informação, Campinas, SP, v. 16, n. 2, mar. 2018. ISSN 1678-765X. Disponível em: <https://periodicos.sbu.unicamp.br/ojs/index.php/rdbci/article/view/8651090>. Acesso em: 26 abr. 2018. doi:https://doi.org/10.20396/rdbci.v16i2.8651090.

PAVÃO, Caterina Groposo; ROCHA, Rafael Porte da; GABRIEL JUNIOR, Rene Faustino. Proposta de criação de uma rede de dados abertos da pesquisa brasileira. RDBCI: Revista Digital de Biblioteconomia e Ciência da Informação, Campinas, SP, v. 16, n. 2, abr. 2018. ISSN 1678-765X. Disponível em: <https://periodicos.sbu.unicamp.br/ojs/index.php/rdbci/article/view/8651180>. Acesso em: 26 abr. 2018. doi:https://doi.org/10.20396/rdbci.v16i2.8651180.

SANTA ANNA, Jorge. Aspectos epistemológicos da ciência da informação e o comportamento informacional: diálogos com Borko, Le Coadic e Saracevic. RDBCI: Revista Digital de Biblioteconomia e Ciência da Informação, Campinas, SP, v. 16, n. 2, jan. 2018. ISSN 1678-765X. Disponível em:

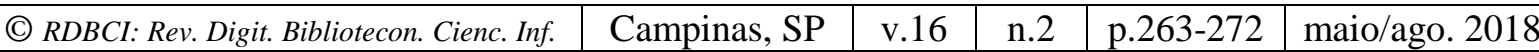


<https://periodicos.sbu.unicamp.br/ojs/index.php/rdbci/article/view/8649807>. Acesso em: 26 abr. 2018. doi:https://doi.org/10.20396/rdbci.v16i2.8649807.

SANTOS, Jacqueline Messias dos. Resenha: Como não publicar artigos. RDBCI: Revista Digital de Biblioteconomia e Ciência da Informação, Campinas, SP, v. 16, n. 2, abr. 2018. ISSN 1678-765X. Disponível em:

<https://periodicos.sbu.unicamp.br/ojs/index.php/rdbci/article/view/8652333 > . Acesso em: 26 abr. 2018. doi:https://doi.org/10.20396/rdbci.v16i2.8652333.

SANTOS, Jovenilda Freitas dos; LUBISCO, Nídia Maria Lienert. O desenvolvimento do marketing e sua aproximação com os paradigmas da Ciência da Informação. RDBCI:

Revista Digital de Biblioteconomia e Ciência da Informação, Campinas, SP, v. 16, n. 2, jan. 2018. ISSN 1678-765X. Disponível em:

<https://periodicos.sbu.unicamp.br/ojs/index.php/rdbci/article/view/8649765>. Acesso em: 26 abr. 2018. doi:https://doi.org/10.20396/rdbci.v16i2.8649765.
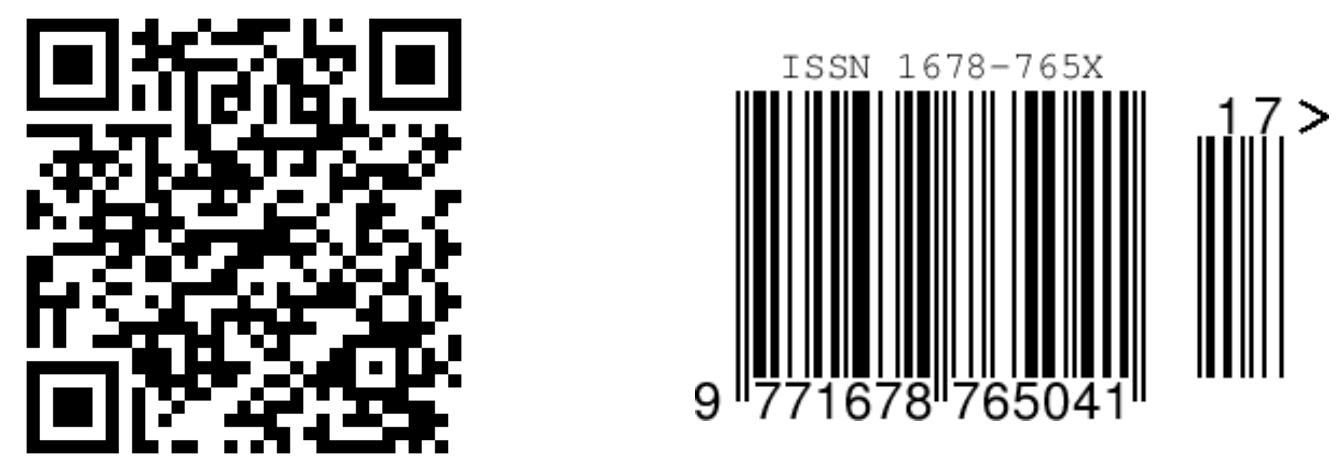